\title{
Enunciación
}

http://revistas.udistrital.edu.co/ojs/index.php/enunc

\section{La lectura de textos clásicos fenomenológicos en la formación de arquitectos}

\author{
Reading of Classical Texts on Phenomenology in Architectural Education \\ Juan Gabriel Ocampo Hurtado ${ }^{1}$
}

Para citar este artículo: Ocampo, J. C. (2014). La lectura de textos clásicos fenomenológicos en la formación de arquitectos. Enunciación, 19(1), 93-106.

Recibido: 15-mayo-2014 / Aprobado: 8-julio-2014

\section{Resumen}

A partir de la investigación sobre la enseñanza de la arquitectura en Colombia llevada a cabo por Alberto Saldarriaga Roa (2011), se plantea la importancia de mejorar el aprendizaje teórico mediante la lectura. Dicho planteamiento motivó la aplicación del método fenomenológico-hermenéutico propuesto por Van Manen a las actividades de cuatro grupos del seminario en Poética y Arquitectura realizado en la Universidad Nacional de Colombia, Sede Manizales. Se encontraron fallas en la enseñanza de la arquitectura provenientes del modelo pedagógico tradicional usado en la generalidad de programas de arquitectura del País, que hacen poco uso de la lectura en la formación académica. Se propone la lectura de textos clásicos sobre fenomenología como didáctica aplicada a la arquitectura a fin de fortalecer el entendimiento y por ende la capacidad de juicio y crítica de los estudiantes.

Palabras clave: aprendizaje teórico, hermenéutica, modelo pedagógico, capacidad crítica

\begin{abstract}
Starting from the research on teaching of architecture in Colombia conducted by Alberto Saldarriaga Roa (2011), the importance of improving theoretical learning through reading is raised. This approach led to application of the phenomenological hermeneutical method proposed by Van Manen to activities of four groups in a seminar of Poetics and Architecture held at the National University of Colombia, Manizales headquarters. Flaws were found in the teaching of architecture from traditional pedagogical models used in most of Colombian architecture programs, which make little use of reading in education. It is proposed that the reading of classic texts on phenomenology as an applied didactic in architecture to strengthen the understanding and therefore judgment and criticism of the students.
\end{abstract}

Keywords: theoretical learning, hermeneutics, educational model, critical capacity

1 Doctor en arquitectura, diseño y urbanismo por la Universidad Autónoma del Estado de Morelos (México); profesor asociado en dedicación exclusiva de la Universidad Nacional de Colombia, Sede Manizales. Correo electrónico: jgocampoh@unal.edu.co 


\section{INTRODUCCIÓN}

\section{La carencia de lectura de naturaleza epistémica como problema del aprendizaje de la arquitectura}

A lo largo del siglo XX la enseñanza de la arquitectura en Colombia tuvo como enfoque pedagógico el modelo conductual que atendía a la relación entre estímulo y respuesta, herencia de la relación entre maestro y aprendiz en su connotación medieval. Como modelo pedagógico tradicional, la transmisión de información de maestro a aprendiz, o de docente a estudiante, aún en el presente, lleva en muchos casos a precisar como objeto central de la enseñanza al taller de diseño arquitectónico. La complejidad del proceso proyectivo y la seducción del trabajo creativo sobre el ejercicio intelectual profundo, condujo en muchos casos a la sobre valoración de la forma, comparada con el ejercicio del juicio y la crítica. La debilidad que surge de una pobre relación entre la teoría y la práctica deja como resultado un bajo nivel cultural para los arquitectos que dan forma a las ciudades colombianas, e incluso latinoamericanas. La lectura, como lo afirma Lomas (2003, p.62), es la interacción entre un lector, un texto y un contexto. Dicha interacción lleva al entendimiento y por tanto a la elevación del juicio. Pero ¿qué tipo de textos puede fortalecer la estructura cognitiva de los arquitectos? En este punto es importante señalar que la arquitectura tiene una fuerte relación con la experiencia sensorial humana. La calidad del habitar humano transita por los órganos sensoriales hasta llegar al nivel inferencial donde la lectura teje su estructura analítica. La consciencia del error propio como parte del proceso derivado de la lectura fortalece la evolución metacognitiva (Pinzás, 2003, p.24). Es por ello que la fenomenología, como enfoque filosófico basado en la percepción sensorial, se plantea como una ruta legítima para la enseñanza y el aprendizaje de la arquitectura. Dando así, aplicación en la arquitectura a la reducción, análisis e interpretación como pasos del análisis fenomenológico.

\section{La lectura del texto fenomenológico como objetivo de enseñanza}

El objetivo de esta investigación es proponer la lectura como medio para la estructuración de la enunciación a partir del desarrollo de los procesos cognitivos de los arquitectos. Ello implica la ruptura de paradigmas pedagógicos, tales como el método de ensayo y error, la elevación de la consciencia urbana como objetivo de la formación y la elaboración de propuestas de orden didáctico orientadas a la formación de arquitectos. La ruptura de paradigmas tiene por objeto considerar la experiencia adquirida por el estudiante de una forma externa a la universidad, como parte integral del ser, de tal forma que el origen del estudiante forme parte de su desarrollo profesional y como tal intervenga en el proceso inferencial (Sánchez y Morales, 2012, p.86). La consciencia sobre la diferencia existente en la estructura de pensamiento de cada estudiante que proviene de un desarrollo cognitivo individual, requiere de quien enseña el respeto por su identidad como estudiante individual. Y ello implica la guía, compañía y enseñanza, pero sobre todo, la cautela ante la posibilidad de la influencia negativa en el campo cognitivo-conductual, de tal modo que los profesores no caigan en la manipulación de los grupos de estudiantes que dirigen.

La búsqueda de lo esencial en la arquitectura tiene que ver con la relación entre el texto y el contexto. La elaboración de interpretaciones a las formas arquitectónicas y los fenómenos o cosas requieren del juicio y la crítica para su construcción. Ello implica la toma de decisiones frente al sentido del arquitecto en contacto con el mundo. Este libre albedrío se refiere a la relación entre la arquitectura, el yo, el otro y el mundo. Para ello el arquitecto elige de una 
forma consciente o inconsciente su enfoque, que puede ser sociológico, artístico, filosófico, psicológico, histórico, científico o tecnológico, por mencionar algunas alternativas; a partir de las cuales elabora sus juicios. No obstante, dichos enfoques tienen fuertes raíces en la estructura cognitiva de los estudiantes. Razón por la que es importante apoyarlos en el proceso de la elevación de su capacidad de juicio, mediante el fortalecimiento de sus procesos racionales que les permitan discernir sobre lo esencial en la arquitectura. Y para ello, ineludiblemente, se debe recurrir a la lectura, ya que conduce al enriquecimiento del intelecto.

Uno de los problemas de la enseñanza centrada en la transmisión de conocimientos es que solo llega al estudiante una porción de la información que necesita para aprender (Carlino, 2005, p.12). Organizar mentalmente lo leído para satisfacer objetivos propios es una actividad de orden cognitivo que obliga al cerebro a estructurar los argumentos.

Si la búsqueda de lo esencial es importante cuando se trata de procesos de transformación de la arquitectura, y por ende del habitar humano, entonces la investigación sobre el sentido de las cosas que afectan la experiencia humana tiene que ver con la arquitectura como texto que se encuentra en el mundo como contexto.

A partir de lo anterior, la elaboración de propuestas de orden didáctico orientadas a la educación y formación de arquitectos tiene en la historia de la fenomenología un amplio y profundo escenario de estudio e investigación, a partir del cual se pueden diseñar nuevas propuestas didácticas, donde la lectura del texto fenomenológico en conjunción con la percepción sensorial se den como alternativas para la elevación de la capacidad del juicio y la crítica.

Así, se plantea la posibilidad de entender a la arquitectura como fenómeno. $Y$, por ende, la utilización de la fenomenología como método que permite encontrar en las visiones de Husserl (1962), Heidegger (1927, 1970), Merleau-Ponty
(1945, 1948), Lévinas (1993), Ricœur (2001) y Derrida (1986) alternativas para el entendimiento de su sentido o el del ser humano como arquitecto. La lectura de textos clásicos fenomenológicos toma forma y señala a los anteriores filósofos como fuente primaria destinada al fortalecimiento de la estructura cognitiva de los estudiantes, ya que su estudio aporta elementos de juicio a partir de los cuales se pueden elaborar juicios y argumentos que fortalezcan teóricamente sus propuestas arquitectónicas.

\section{Entre el Proyecto y el Pensamiento}

La evaluación del rendimiento de estudiantes universitarios sometidos a los exámenes del Estado Saber Pro y Ilevados a cabo por el ICFES ha demostrado sistemáticamente la deficiencia en competencias lingüísticas. En el campo de la arquitectura el tema es crítico. Si bien la preocupación por la crisis de la enseñanza de la arquitectura en Colombia (Saldarriaga, 2011) ha obligado a tomar medidas severas en muchas de las universidades colombianas, persiste en muchos casos la debilidad en el uso de la lectura como medio de aprendizaje.

En la rigurosa investigación realizada por Saldarriaga Roa (2011) y publicada por la Asociación Colombiana de Facultades de Arquitectura se presenta el porcentaje de dedicación a diez áreas del conocimiento. Dicho análisis se realizó a 33 programas colombianos de arquitectura. En lo planteado por Saldarriaga Roa las áreas temáticas más significativas con respecto a la lectura y escritura son la de Proyecto y la de Pensamiento. La primera, por ser históricamente el eje de formación de todos los programas de arquitectura y contener elementos de referencia lingüístico-textual y la segunda por incluir asignaturas como historias y teorías. Poner en contraste los porcentajes de dedicación a cada una de estas áreas significa visualizar el énfasis en el conocimiento práctico en relación con el conocimiento teórico. Los datos de Saldarriaga Roa son los siguientes: Tabla 1. 
Tabla 1. Relación del porcentaje de dedicación de las universidades a Proyecto y Pensamiento

\begin{tabular}{|c|c|c|c|}
\hline & Universidad & $\%$ dedicado a Proyecto & $\%$ dedicado a Pensamiento \\
\hline 1 & Autónoma del Caribe & 37,65 & 13,53 \\
\hline 2 & Del Atlántico & 29,35 & 13,59 \\
\hline 3 & C.U. de la Costa & 27,78 & 9,26 \\
\hline 4 & De América & 34,18 & 8,86 \\
\hline 5 & De los Andes & 40,37 & 9,32 \\
\hline 6 & Antonio Nariño & 36,78 & 14,94 \\
\hline 7 & Católica de Colombia (Bog.) & 29,22 & 15,58 \\
\hline 8 & La Gran Colombia & 32,06 & 9,16 \\
\hline 9 & Pontificia U. Javeriana & 40,43 & 9,57 \\
\hline 10 & Jorge Tadeo Lozano (Bog.) & 68,72 & 19,43 \\
\hline 11 & Nacional de Colombia (Bog.) & 41,04 & 15,67 \\
\hline 12 & Piloto de Colombia & 33,94 & 6,06 \\
\hline 13 & Antonio Nariño (Bog.) & 37,71 & 14,86 \\
\hline 14 & Santo Tomas & 32,10 & 10,49 \\
\hline 15 & San Buenaventura & 56,58 & 9,87 \\
\hline 16 & Del Valle & 41,42 & 8,88 \\
\hline 17 & Jorge Tadeo Lozano (Cartagena) & 27,78 & 30,00 \\
\hline 18 & De Ibagué & 46,43 & 15,48 \\
\hline 19 & Católica de Manizales & 22,86 & 5,71 \\
\hline 20 & Nacional de Colombia (Mzls). & 35,59 & 8,47 \\
\hline 21 & Nacional de Colombia (Med.) & 32,27 & 8,70 \\
\hline 22 & Pontificia Bolivariana (Med.) & 31,87 & 9,89 \\
\hline 23 & Del Sinú & 35,40 & 17,39 \\
\hline 24 & Pontificia Bolivariana (Montería) & 50,00 & 9,68 \\
\hline 25 & CESMAG (Pasto) & 39,52 & 11,38 \\
\hline 26 & De Nariño & 34,25 & 11,60 \\
\hline 27 & Católica de Risaralda & 36,53 & 13,77 \\
\hline 28 & De Boyacá & 31,95 & 9,47 \\
\hline 29 & Sto. Tomás (Tunja) & 26,71 & 22,98 \\
\hline 30 & Del Pacífico (Buenaventura) & 26,49 & 18,54 \\
\hline 31 & San Buenaventura (Cartagena) & 51,85 & 11,11 \\
\hline 32 & Sto. Tomás (Medellín) & 37,35 & 12,05 \\
\hline 33 & C.U. del Caribe (Sincelejo) & 33,93 & 11,90 \\
\hline
\end{tabular}

Fuente: Saldarriaga, 2011.

A partir de los anteriores datos se podrían hacer las siguientes interpretaciones:

Los planteamientos realizados por varios arquitectos en la revista HITO de agosto de 2002, dedicada a la enseñanza de la arquitectura, según los cuales la gran mayoría de programas curriculares de arquitectura del país tenían como eje central al proyecto eran ciertos (Ocampo, 2013). En dicha revista se presentaba la preocupación por la concentración del perfil profesional en el 
diseño arquitectónico y el bajo nivel de dedicación profesional a actividades como la construcción, administración e investigación. Algo más que se puede afirmar es que el $97 \%$ de los programas de arquitectura mencionados por Saldarriaga Roa dedican mayor porcentaje al Proyecto en comparación con el área de Pensamiento. El único programa que tiene un comportamiento contrario a esta tendencia es el de la Universidad Jorge Tadeo Lozano de Cartagena.

El área de proyecto tiene el siguiente comportamiento:

Promedio: $36,973 \%$ Tendencia: $37,4168 \%$

La dispersión señala al programa de la Universidad Jorge Tadeo Lozano de Bogotá con un comportamiento especial dentro del conjunto de datos presentes.

El área de Pensamiento tiene el siguiente comportamiento:

Promedio: 12,642\% Tendencia: 11,821\%

La dispersión señala al programa de la Universidad Jorge Tadeo Lozano de Cartagena con un comportamiento especial dentro del conjunto de datos presentes.

Es conveniente señalar que la participación del área de Pensamiento se encuentra entre el $5,71 \%$ y el $30,00 \%$, con una tendencia al $11,821 \%$ como tiempo dedicado a las asignaturas del conocimiento teórico.

De forma adicional, se ha realizado una búsqueda en los sistemas de ranking internacional para definir los programas nacionales que se encuentren incluidos en la lista de Saldarriaga Roa y proceder a hacer una comparación con los programas señalados por la dispersión. Los programas resultantes fueron: Pontificia Universidad Javeriana, Universidad de Los Andes y Universidad Nacional de Colombia, Sede Bogotá. La fuente de lo anterior se obtuvo en Graduate Architecture (2014), University Rankings, (2014) y 4 International Colleges \& Universities (2014).

Las cinco universidades seleccionadas para analizar la relación entre la dedicación al área de Proyecto y al área de Pensamiento son las siguientes: Tabla 2.

A partir de estos datos se puede decir lo siguiente:

Tabla 2. Universidades seleccionadas para analizar la dedicación al área de Proyecto y al área de Pensamiento

\begin{tabular}{|c|c|c|c|}
\hline & Universidad & $\%$ dedicado a Proyecto & $\%$ dedicado a Pensamiento \\
\hline 1 & Universidad de Los Andes (Bog.) & 40,37 & 9,32 \\
\hline 2 & Pontificia Universidad Javeriana (Bog.) & 40,43 & 9,57 \\
\hline 3 & Universidad Jorge Tadeo Lozano (Bog.) & 68,72 & 19,43 \\
\hline 4 & Universidad Nacional de Colombia (Bog.) & 41,04 & 15,67 \\
\hline 5 & Universidad Jorge Tadeo Lozano (Cartagena) & 27,78 & 30,00 \\
\hline
\end{tabular}

Fuente: elaboración propia. 
- Tres de estas universidades tienen una dedicación al área de Proyectos con un porcentaje cercano al $40 \%$, lo cual las ubica por encima del promedio y de la tendencia. Estas tres universidades tienen excelente calificación en sistemas mundiales de ranking.

- De estas tres universidades, dos presentan la menor dedicación porcentual al área de Pensamiento, cercano al $9 \%$ y ambas universidades son privadas. Este comportamiento se encuentra por debajo del promedio y de la tendencia.

- La Universidad Jorge Tadeo Lozano de Bogotá presenta el mayor porcentaje de dedicación al área de Proyectos debido a que tiene un diseño curricular que busca la formación integral a partir de dicha área, de acuerdo con la información suministrada por Saldarriaga Roa. Tiene, además, una dedicación de 19,43\% al área de Pensamiento, valor superior al de las tres universidades mejor calificadas en ranking mundial.

- La única universidad que tiene un mayor porcentaje de dedicación al área de Pensamiento en comparación con el área de proyecto no está calificada en posiciones significativas de ranking internacional. No obstante, la proporción presente en este currículo podría llevar a formar arquitectos con una elevada capacidad de juicio y crítica.

De acuerdo con lo anterior se llega a las siguientes interpretaciones:

La similitud en la dedicación de la Universidad de los Andes, la Pontificia Universidad Javeriana y la Universidad Nacional de Colombia, las ubica en un nivel de excelencia semejante, a partir del cual se definen diferencias curriculares según la legítima particularidad de los perfiles profesionales buscados.

La propuesta curricular de la Universidad Jorge Tadeo Lozano de Bogotá es innovadora, dado su interés por la formación integral donde el área de Proyecto rebasa lo proyectivo y llega hasta otros territorios como el conceptual. Esto, sumado a la dedicación adicional al área del Pensamiento, dejará ver posiblemente excelentes resultados en el futuro.

La re-estructuración de currículos frente a la crisis de la enseñanza de la arquitectura en Colombia ha llevado a la búsqueda de alternativas relacionadas con las particularidades de cada universidad. Se esperaría que el programa con mayor dedicación a las asignaturas teóricas tuviera mayor reconocimiento mundial, pero esto no ocurre. Resulta entonces una pregunta de valor sustantivo: ¿por qué la mayor dedicación de tiempo al área de Pensamiento en comparación con el área de Proyecto no garantiza la calidad del programa?

La respuesta se puede encontrar en el modelo pedagógico y en el tipo de didácticas utilizadas. Es posible que la Universidad Jorge Tadeo Lozano de Cartagena tenga las mismas dificultades presentes en un alto porcentaje de los programas universitarios del país, que es el de la lectura y escritura. Si esta situación fuera así, la propuesta de dicha universidad, que apunta a formar arquitectos con un agudo nivel de juicio y donde la relación entre profesor y estudiante es estrecha (Carrasco, 2004), solo podría ser posible a partir del fortalecimiento en competencias referentes a la lectura y la escritura donde estudiantes y profesores tomen parte activa del proceso. Una forma de hacerlo es mediante el tipo de actividades que se llevan a cabo en torno a la lectura. La actividad que se da al tratar de comprender un texto es responsable del aprendizaje aunque este no sea el propósito de la lectura (Solé, 1992). De una forma que solo la comunidad académica de la Universidad Jorge Tadeo Lozano de Cartagena podría plantear, las actividades que se pueden derivar de su currículo se encuentran en relación directa con las condiciones del lugar como hecho cultural.

El tiempo y los créditos curriculares existen en las diferentes propuestas académicas. Pero, ¿se enseña un conocimiento?, ¿se está fortaleciendo la capacidad de juicio? La evolución cognitiva de los estudiantes puede revelar esa diferencia y ello se aprecia en el nivel de discusión y riqueza cultural de los estudiantes o profesionales. La carencia de 
la lectura no fundamenta la construcción de argumentos. Si el propósito como formadores de arquitectos es el de proteger el contexto urbano actual y futuro, entonces valdría la pena retomar la lectura como didáctica para fortalecer la evolución cognitiva de los estudiantes en aras de proveerles de un mayor nivel de juicio y de crítica.

\section{El texto fenomenológico}

Si la lectura favorece la capacidad de entendimiento (Lomas, 2003, p.58), leer sobre las formas de entender un contexto puede ser útil para que un estudiante mejore sus competencias al tratar de comprender el problema arquitectónico.

Según la Real Academia Española de la Lengua, la fenomenología es la teoría de los fenómenos o de lo que aparece. También lo define como el método filosófico desarrollado por Edmund Husserl que, partiendo de la descripción de las entidades y cosas presentes a la intuición intelectual, logra captar la esencia pura de dichas entidades trascendente a la misma consciencia.

Bajo la primera acepción se propone entender a la arquitectura como fenómeno donde este significa toda manifestación que se hace presente a la consciencia de un sujeto y aparece como objeto de su percepción o, en la filosofía de Immanuel Kant, lo que es objeto de la experiencia sensible (RAE). De acuerdo con esto, la arquitectura sería una manifestación que es percibida por un sujeto. Lo cual es cierto. Dado que la arquitectura puede así ser entendida como fenómeno entonces podría ser materia de estudio de la fenomenología.

Por otra parte, cuando Husserl define a la fenomenología como método filosófico, señala la importancia del ejercicio intelectual sobre la descripción de las entidades y cosas presentes. Ello establece una relación entre el sujeto que habita y lo que percibe. Dicho planteamiento ubica al individuo en el espacio en actitud perceptiva y dispuesto a la inferencia.

Ya que fenómeno y fenomenología se encuentran en relación directa con la arquitectura, entonces la esencia y el sentido de la misma podrían ser objeto de estudio a partir de la percepción sensorial. Pero para ello sería inevitable la lectura de los textos clásicos fenomenológicos.

El reto de estudiar la arquitectura a partir del enfoque fenomenológico es mayúsculo para cualquier persona, ya que implica el estudio de planteamientos filosóficos como los de Husserl, Heidegger, Merleau-Ponty, Lévinas, Ricœur y Derrida. Ello en otras palabras significa el estudio de la historia de la filosofía del siglo XX.

Edmund Husserl, fundador de la fenomenología trascendental, plantea la reducción fenomenológica sobre la percepción sensorial del objeto en búsqueda de la esencia fundamental. Husserl diferencia entre lo inmanente y lo trascendente en esta búsqueda. Es decir, entre lo que es inseparable de la esencia del ser y lo que está más allá de los límites de dicha esencia. Desde el punto de vista de la arquitectura, la búsqueda de esta esencia se encuentra entre la forma y la estructura como lo inmanente y el contenido como lo trascendente. Este autor menciona:

El yo como persona, como cosa del mundo, y la vivencia como vivencia de esta persona, insertados ambos, aunque sea de un modo indeterminado, en el tiempo objetivo, todo ello son transcendencias $y$, como tales, gnoseológicamente son cero. Solo por medio de una reducción —a la que vamos también a llamar ya reducción fenomenológica- obtengo un dato absoluto, que ya no ofrece nada de trascendencia. (Husserl, 1970, p.20)

La objetividad que plantea Husserl no intenta hacer caso omiso de lo trascendente, sino determinar lo inmanente manteniendo la consciencia sobre la existencia de lo trascendente (Husserl, 1962). Se puede analizar así la forma en condiciones objetivas tal como ocurrió en la Bauhaus en Alemania con arquitectos como Bruno Taut, Hans Poelzig o Erich Mendelsohn, quienes reaccionaron ante el expresionismo mediante el movimiento de la nueva objetividad, que tuvo como base una visión más práctica de la arquitectura. 
Martin Heidegger, por su parte, plantea la fenomenología hermenéutica como propuesta investigativa mediante la que se puede elaborar la interpretación del ser en el "ahí". En este caso, la existencia del habitante como ser en una circunstancia donde confluyen los verbos ser y estar implican que el habitante "es" en el sitio, pero también "está". La forma de ser y estar responde, de acuerdo con la interpretación que Derrida da al discurso de Platón sobre khôra, a la noción de lugar. Esto implica la aceptación del contexto temporal, por lo que historia, cultura, filosofía o lingüística son dimensiones válidas (Heidegger, 1927 y 1970) que convergen en el ser consciente como habitante del lugar.

Maurice Merleau-Ponty en sus Conversaciones de 1948 presentó sus siete conferencias sobre el mundo de la percepción, en donde plantea, entre otras cosas, al espacio como exploración del mundo percibido. En este documento afirma:

Todo cambia cuando, con las geometrías Ilamadas no euclidianas, se llega a concebir el espacio como una curvatura propia, una alteración de las cosas por el solo hecho de su desplazamiento, una heterogeneidad de las partes del espacio y de sus dimensiones que dejan de ser sustituibles una por otra y afectan a los cuerpos que en él se desplazan con ciertos cambios. (Merleau-Ponty, 1948, p.18)

La consciencia de la alteración del sentido de la arquitectura a partir de la modificación en la ubicación de los elementos que la constituyen implica la búsqueda de la relación entre el texto y el contexto (Merleau-Ponty, 1945 y 1948). Esta relación se busca en el sentido, que tiene una forma determinada en uno u otro contexto. A modo de ejemplo: ¿por qué las cubiertas en el período gótico alemán tenían alta pendiente?, ¿por qué, por otra parte, las cubiertas de la ciudad estado de Xochicalco, pertenecientes al período Epiclásico mesoamericano, eran planas? (Ocampo, 2009). Estas particularidades fueron el resultado de respuestas técnicas a circunstancias climáticas y culturales de su tiempo y lugar. Es decir, a la relación entre texto y contexto.

Emmanuel Lévinas resalta la alteridad como forma de entendimiento del yo a partir del respeto por el otro. La intersubjetividad planteada como comunicación intelectual entre varios sujetos puede ser estudiada como la relación entre diferentes formas de entender o expresar la arquitectura; entre las que se podría mencionar al enfoque social en contraste con el enfoque netamente plástico o formal. El hombre como ser temporal puede habitar la arquitectura en una experiencia de reconocimiento hacia la otredad. De esta forma, la relación entre el otro, yo y la arquitectura, plantea un sentido de la existencia (Lévinas, 1993).

Paul Ricœur (2001) Ileva la fenomenología hasta la hermenéutica donde reivindica la interpretación. Con esto ofrece rutas metódicas para la investigación en arquitectura. En su visión la relación entre tiempo, espacio y narración es compatible de una forma fluida con la arquitectura. En este caso el recorrido físico de la obra, ya sea de escala arquitectónica o urbana, implica inicio, desarrollo del tema y conclusión. El acto de desplazarse desde un punto a otro, desde una plaza urbana hasta otra, supone el paseo por sitios llenos de historias que acompañan la experiencia poética de caminar. La narración de la arquitectura, o la arquitectura como objeto narrativo, puede ser experimentada de una forma secuencial tal como el objeto literario. Aristóteles en su Poética podría ser llevado a la arquitectura a partir de la interpretación hermenéutica de Ricœur. La relación entre obra, arquitecto y arte (Aragón, 2009, p.62) o la peripecia y el reconocimiento como temas de composición arquitectónica establecen formas de entender la arquitectura.

Jacques Derrida busca deconstruir lo analizado hasta llegar a su esencia fundamental.

Cuando describo el fenómeno, no describo la cosa en sí misma, por así decirlo, más allá de su aparecer, sino su aparecer para mí, tal como se me aparece. ¿Con qué me las tengo que ver en cuanto la cosa se me aparece? Se trata de una operación 
muy delicada, pues resulta muy difícil disociar la realidad de la cosa del aparecer de esa cosa. (Spire, 1999)

Derrida señala que la evolución de la fenomenología aún no concluye y, por el contrario, es un escenario útil para investigaciones futuras. De hecho, el deconstructivismo, movimiento arquitectónico que nació a fines de la década de los ochenta, ha sido tomado como enfoque para la reflexión arquitectónica en diversos escenarios mundiales (Derrida, 1986). Obras como las de Frank Gehry, con su Museo Guggenheim de Bilbao, o como las de Peter Eisenman con su Ciudad de la cultura de Galicia, demuestran investigación en la forma como se aparece la obra a su habitante. Como lo percibe e interpreta su sentido.

Las anteriores consideraciones indican el valor de la lectura de los textos clásicos fenomenológicos en la enseñanza y aprendizaje de la arquitectura, puesto que su utilidad radica en la potencial evolución cognitiva de los estudiantes a partir del fortalecimiento del entendimiento de sus objetos y temas de estudio. Para ello se propone la enseñanza y el aprendizaje a partir de la lectura como didáctica.

\section{METODOLOGÍA}

Max Van Manen, profesor emérito de la Universidad de Alberta, ha dedicado su labor investigativa a la aplicación de la fenomenología en la educación. Es considerado como el pionero en la fenomenología hermenéutica como metodología de investigación educativa (Ayala, 2008, pp.409-430). Su método fue empleado en un periodo de dos años en los que cuatro grupos del Seminario de profundización en Arquitectura y Poética de la Universidad Nacional de Colombia, Sede Manizales, fueron expuestos de una forma consciente a la lectura de textos de orden fenomenológico. Este seminario se dicta en noveno semestre del programa de arquitectura.
Como primera etapa de la investigación, se dio la recopilación de información teórica. Dicha recopilación se llevó a cabo a partir de la tensión producida por discusiones y debates de los grupos. Se buscó reunir un conjunto de afirmaciones provenientes de los estudiantes que se refirieran a su experiencia. Cada correlato de los estudiantes transforma la percepción general a partir de las incertidumbres y vacíos presentados (Iser, 1987, p.151). Ello significa que el conjunto de ideas provenientes de los estudiantes revelan el mismo contexto de formación, pero para personas que evidentemente tienen orígenes totalmente diferentes. Con ello la búsqueda de objetividad en el conjunto de datos lleva, a la postre, a la propuesta de resultados que pueden beneficiar al currículo. La entrevista conversacional y la observación directa propuestas por Van Manen fueron actividades de orden estructural que dieron paso a la reducción fenomenológica, que tiene por objeto el entendimiento de la experiencia.

En esta etapa, la enunciación del juicio de cada miembro de grupo sobre el sentido de su rol como estudiante de arquitectura llevó a la catarsis a modo de terapia grupal, en donde cada quien expresó libremente su estado personal con respecto a su formación como arquitecto. Para ello, la entrevista conversacional a cada estudiante y la observación directa, llevada a cabo por el profesor, sirvieron como actividad didáctica para alentar la discusión de grupo. A pesar de haberse realizado la recolección de información en cuatro semestres diferentes, ya que la duración de cada curso era de un semestre, las respuestas de los individuos fueron similares. Se dieron expresiones como las siguientes:

"A mí lo que me interesa es la construcción. No quiero volver a diseñar en mi vida".

"Yo quiero dedicarme totalmente al diseño".

"No quiero diseñar ni construir. No sé qué hacer".

Las expresiones reunidas conformaron el primer conjunto de datos a partir del cual se llevaría a cabo el análisis de la investigación. En los cuatro 
grupos estudiados el comportamiento de los individuos y los datos obtenidos fue similar.

Luego de estas discusiones, los grupos fueron expuestos a lecturas de orden fenomenológico en donde el entendimiento de la arquitectura a partir del uso consciente de los órganos sensoriales fue el eje fundamental. Como ejercicio práctico, los estudiantes debían elegir un sentido a partir del cual dar énfasis en su análisis de la arquitectura. Si un estudiante optaba por el sentido del olfato, debía realizar la búsqueda de lecturas en las que se relacionara la arquitectura con ese tipo de sensaciones y proceder a hacer la exposición oral respectiva en las discusiones de grupo. Al finalizar esta actividad los estudiantes hicieron el siguiente tipo de afirmaciones:

"Esto lo deberían enseñar en primer semestre y no en noveno".

"Por qué no nos contaron esto antes".

"El uso integral de los sentidos mejora el entendimiento del lugar".

Con este segundo conjunto de expresiones se consolidó la base de datos fundamental requerida por el método de Van Manen. A partir de allí se procedió con el análisis o reducción fenomenológica.

En la reducción, o segunda etapa, se buscó la importancia pedagógica de la experiencia (Van Manen, 2003). Se tuvo como objetivo la determinación de la esencia que contiene el conjunto de datos obtenidos. Las reflexiones y discusiones de grupo sobre este tema pusieron en contraste el modelo de educación tradicional con la importancia del entendimiento como factor presente en la evolución cognitiva. La dificultad inicial de los ejercicios de lectura magnificó el contraste entre el tiempo dedicado al área de Proyecto y al área de Pensamiento. Si bien, el tiempo dedicado a las asignaturas de historia fue adecuado, el bajo nivel de lectura en el presente fue coherente con la inexistencia de esta actividad como didáctica a lo largo del programa vivido en el pasado. Los estudiantes manifestaron el uso mínimo de la lectura a lo largo de los últimos cuatro años. Ello indica falencias en dicha actividad como parte de la didáctica.

A lo largo de los ejercicios de reducción los argumentos provenientes de la emoción y de la subjetividad dieron paso a enunciados con acuerdos de grupo, donde se hicieron evidentes situaciones que podrían ser mejoradas en el currículo. Los siguientes son los enunciados fundamentales resultantes del análisis:

El currículo de arquitectura al que pertenecen los estudiantes está orientado al perfil de arquitecto como diseñador.

Este currículo no hace énfasis en perfiles profesionales como la construcción, la administración o el urbanismo.

Este currículo carece en un alto grado de procedimientos didácticos basados en la lectura o escritura.

Los estudiantes de esta carrera desconocen el significado de la fenomenología.

Al llegar a un nivel de entendimiento diferente al obtenido a lo largo de la carrera, cada miembro de grupo replanteó o reafirmó su posición frente al proceso de formación que ha vivido. Esto significó la elevación de la consciencia sobre su existencia en ese momento y en el mundo. Para fortalecer dicho objetivo cognitivo, se procedió con la tercera etapa de la investigación, la escritura.

La tercera etapa investigativa corresponde a la escritura de un texto de orden fenomenológico en donde se estructuró la exposición oral presentada inicialmente, pero enriquecida con las discusiones de grupo. De esta forma, la didáctica de la lectura trasciende a la demostración del entendimiento de ideas a partir de la escritura. La presentación en público de las ideas resultantes permitió el ejercicio de la argumentación y discusión como actividad diferente a lo acostumbrado en esta carrera, que es la elaboración de planos arquitectónicos.

Los estudiantes expresaron su dificultad frente al reto de la escritura ya que fue muy poco el uso de este medio a lo largo de su carrera para 
comunicar las ideas. También expresaron el bajo nivel de lectura que tuvieron durante ese período.

\section{PROPUESTAYANÁLISISDE RESULTADOS}

Las lecturas y discusiones de los seminarios dieron como resultado la toma de consciencia sobre la importancia de los textos clásicos fenomenológicos en relación con la arquitectura. Ello implica el enriquecimiento de las reflexiones y discusiones en torno a las formas de enseñar o aprender. La labor de enseñanza, que es muy importante, puede tener efectos profundos en la estructura cognitiva de los estudiantes a partir de los elementos de juicio y la exposición al criterio del profesor, la cual es recibida como idea valiosa ¿Qué ocurre cuando el profesor se excede en su capacidad de transformar la conducta de sus estudiantes? Quien enseña está en capacidad de hacer esto por el simple hecho de actuar en el rol de maestro ¿Qué tan preparados están los profesores universitarios colombianos en pedagogía y didáctica? Las fuertes exigencias para ingresar a la carrera docente no siempre incluyen formación en pedagogía ¿Cómo enseña entonces la generalidad de profesores? Se podría afirmar que como aprendieron. Es decir, como vieron que les enseñaron sus propios profesores. Pero el tema de la educación es de vital importancia para el desarrollo del país, y es así como conviene retomar el valor de la lectura como estrategia central del aprendizaje y no como actividad periférica al proceso de enseñanza.

La conciencia de los estudiantes, sobre la reflexión anterior, les llevó a analizar sus métodos de análisis y diseño con lo que se dieron discusiones sobre el quehacer actual del arquitecto y su participación en este escenario.

La lectura de textos clásicos fenomenológicos dio pautas para enfrentar el tema de la arquitectura a partir del siguiente tipo de inquietudes:

- La forma arquitectónica no solo responde a un interés plástico sino que tiene otros aspectos que le dan valor o sentido. Para tal efecto se recomienda la lectura de los textos de Edmund Husserl como fundador del movimiento de la fenomenología. La forma pura, libre de aspectos trascendentales, es una alternativa para este tipo de análisis. La orientación didáctica de estos textos se refirió al estudio de la diferencia entre lo inmanente y lo trascendente. La lectura inicial se interpretó de forma grupal desde el ámbito de la arquitectura en búsqueda de su esencia a partir del ejercicio de la interpretación. Se llegó al punto de diferenciar la forma pura del contexto histórico y social, con lo cual se entendieron algunos tipos de expresiones arquitectónicas contemporáneas.

- La arquitectura, como lugar donde es importante la interpretación del hombre como habitante, acepta la relación del texto en el contexto. En este caso, lauras a los textos de Martin Heidegger son apropiadas para la estructuración del estudiante cuando este es su interés. En este caso, la didáctica de la lectura tenía por objetivo entender la relación entre los factores que configuran a la arquitectura como lugar. El vínculo entre el sentido de la obra con su contexto histórico y social es enriquecido por el contenido de la obra. La estética como crítica existencial se plantea como aporte al aprendizaje. Los estudiantes plantearon diferentes formas de enriquecer la estética como crítica de sus planteamientos arquitectónicos.

- Si la búsqueda formal y la alteración al sentido de la arquitectura mediante la variación a los elementos que la conforman es de interés para el estudiante, entonces la profundización en temas de la percepción es una alternativa de estudio legítima. Se recomienda en ese caso el estudio de los textos de Maurice Merleau-Ponty y, a partir de este referente, el análisis a las obras de Juhanni Pallasmaa, Peter Zumthor y Steven Holl, en cuyas propuestas arquitectónicas se aprecia una sensibilidad que supera el omnipresente sentido de la vista para reivindicar las sensaciones captadas por los otros órganos sensoriales.

- Si, por otra parte, el estudiante está interesado en valorar al otro, a los habitantes en relación con su obra, entonces el individualismo es superado 
por la elevación de la conciencia. Se recomienda la lectura de la obra de Emmanuel Lévinas. En este caso, la didáctica se aplicó al estudio de la arquitectura como asunto social. Fue uno de los espacios de discusión con mayor participación, ya que se dieron críticas con respecto a la falta de formación social en comparación con el énfasis formalista presente en muchos de los programas de arquitectura del país.

- La interpretación como alternativa donde tiempo y espacio se conjugan puede plantearse como una dirección de estudio profunda y de amplias posibilidades investigativas. Paul Ricœur se recomienda para el estudio profundo de sus lecturas. La didáctica de la lectura tuvo por objeto el análisis al problema del patrimonio arquitectónico donde la dinámica de la histórica formó parte de las discusiones en las que el tiempo y el espacio formaron parte de la arquitectura. El juicio a la obra arquitectónica con valor histórico se ejercitó en búsqueda de la determinación de validez. Para este ejercicio los estudiantes propusieron obras a fin de ser sometidas al juicio del grupo.

- Por su parte, hay estudiantes que optan por la deconstrucción conceptual de la obra. Por la búsqueda de la esencia fundamental y la eliminación de elementos que no son significativos en el discurso arquitectónico. La lectura a la obra de Jacques Derrida resulta de indiscutible utilidad en este caso. La búsqueda de lo fundamental como objetivo didáctico fue motivo de discusión a partir de las diferencias conceptuales de los estudiantes. No obstante, la actividad del debate a lo largo de estas lecturas fortaleció los argumentos y las estructuras mentales de los estudiantes. Al finalizar cada sesión de discusión se elaboraba una conclusión del proceso vivido con el objeto de entender la existencia de diferentes opiniones frente a los temas estudiados.

\section{CONCLUSIONES}

La existencia de un área del conocimiento denominada por Saldarriaga Roa (2011) como
Pensamiento, tiene cabida en los programas curriculares de Colombia. En este espacio se incluyen asignaturas como historia o teoría. Valdría la pena incorporar en esta franja el estudio a la fenomenología. Ello implica un esfuerzo por parte de profesores en su labor de enseñanza y la adopción de la lectura como actividad central del aprendizaje.

Las deficiencias en lectura y escritura, presentes en un amplio sector de los programas universitarios del país, requieren intervención inmediata. La actualización o formación de los docentes en pedagogía es necesaria a fin de estructurar de una forma legítima la lectura como actividad de aprendizaje en el programa de arquitectura. El beneficio de este tipo de medidas se puede reflejar en el nivel cultural y en la construcción de argumentos de los futuros profesionales.

Una de las formas fundamentales por medio de las cuales los arquitectos adquieren información del mundo es la percepción sensorial. Este contacto sensible ha sido estudiado a nivel profundo por la corriente fenomenológica que podría definirse como la filosofía del siglo XX. La lectura como medio para fortalecer el entendimiento de este tipo de ideas no es fácil para una población que no tiene la costumbre de leer. No obstante, esta dificultad debe ser enfrentada no solo por los estudiantes, sino por los profesores.

La lectura como parte del conocimiento teórico demuestra su importancia en la actividad inferencial donde lo captado por los órganos sensoriales va en conjunción con el aprendizaje razonado. Si la enseñanza de la arquitectura ha tenido un alto componente práctico, como lo demuestra Saldarriaga Roa (2011) y si, además, las asignaturas referentes al pensamiento, es decir, historias o teorías, se encuentran presentes en los currículos, entonces, ¿por qué hay falencias tan fuertes en el campo de la lectura y la escritura? Se evidencia la pedagogía tradicional sobre el uso de la lectura profunda y crítica. Se recomienda, a modo de conclusión, dar mayor protagonismo al estudiante como legítimo actor del proceso de aprendizaje. 


\section{RECONOCIMIENTOS}

Esta investigación se denomina Lógica y Arquitectura, la cual desarrolló el Grupo de Trabajo Académico de Medios de Expresión y Comunicación de la Universidad Nacional de Colombia Sede Manizales. Código HERMES 12579. Fue financiada totalmente por dicha universidad.

\section{REFERENCIAS}

Aragón, G. (2009). El criticismo literario clásico: ¿prefacio de la didáctica de la literatura contemporánea? Enunciación, 14(1), 60-72.

Ayala, R. (2008). La metodología fenomenológico-hermenéutica de $M$. Van Manen en el campo de la investigación educativa. Posibilidades y primeras experiencias. Revista de Investigación Educativa. 26, (2), 409-430.

Carlino, P. (2005). Escribir, leer y aprender en la universidad: una introducción a la alfabetización académica. Buenos Aires: Fondo de Cultura Económica.

Carrasco, J. (2004). Una didáctica para hoy, cómo enseñar mejor. Madrid: RIALP.

Derrida, J. (1995). Khôra. Córdoba: Alción Editora.

Derrida, J. (1986). La metáfora arquitectónica. Recuperado de http://www.jacquesderrida.com.ar/textos/arquitectura.htm Consultado en 14/05/2014

Graduate Architecture (2014). The World's Best Architecture Schools. Recuperado de http://www. graduatearchitecture.com/ARCHSCHOOLS/ archschools_en.html\#Contact

Heidegger, M. (1970). El arte y el espacio. Eco. Revista de la Cultura de Occidente, 21(2), 113-120.

Heidegger, M. (1927). Ser y tiempo. Edición electrónica: www.philosophia.cl Escuela de Filosofía Universidad ARCIS. Recuperado de http://www.magonzalezvalerio.com/textos/ser_y_tiempo.pdf

Husserl, E. (1970). La idea de la fenomenología. Recuperado de http://biblio3.url.edu.gt/Libros/joyce/ idea.pdf

Husserl, E. (1962). Ideas relativas a una fenomenología pura y una filosofía fenomenológica. México - Buenos Aires: Fondo de Cultura
Económica. $2^{\text {a }}$. edición en español. Primera edición en alemán, 1913. Recuperado de https://profesorvargasguillen.files.wordpress.com/2012/11/ husserl-edmund-ideas-relativas-a-una-fenomenologia-pura-y-una-filosofia-fenomenologica-ocr.pdf

ICFES (2013). Resultados agregados Saber Pro 2013. Recuperado de http://www.icfes.gov.co/ resultados/saber-pro-resultados-individuales/ resultados-agregados-2013

Iser, W. (1987). El proceso de lectura: enfoque fenomenológico. En J. A. Mayoral (Comp.) Estética de la recepción. Madrid: Arco.

Lévinas, E. (1993). El tiempo y el otro. Barcelona: Paidós.

Lomas, C. (2003). Leer para entender y transformar el mundo. Enunciación, 8(1), 57-67.

Merleau-Ponty, M. (1945). Fenomenología de la percepción. Barcelona: Península.

Merleau-Ponty, M. (1948). El mundo de la percepción. México: Fondo de Cultura Económica.

Ocampo, J. (2013). Didáctica y percepción de la arquitectura. HITO, 27.

Ocampo, J. (2009). El lugar de la ciudad arqueológica, Xochicalco (Tesis de doctorado). Universidad Autónoma del Estado de Morelos, México.

Pinzás, J. (2003). Metacognición y lectura. Lima: Pontificia Universidad Católica del Perú.

Platón (1872). Obras completas, 6, Timeo. Madrid: Patricio de Azcárate (Ed.). Recuperado de http:// www.filosofia.org/cla/pla/img/azf06131.pdf

QS University Rankings (2014). Top Ten Universities in Latin America 2013. Recuperado de http://www.topuniversities.com/university-rankings-articles/latin-american-university-rankings/ top-ten-universities-latin-america-2013

Ricœur, P. (2001). Del texto a la acción: ensayos de hermenéutica. México: Fondo de Cultura Económica.

Saldarriaga, A. (2011). Enseñanza de la arquitectura en Colombia, estado actual. Asociación Colombiana de Facultades de Arquitectura.

Sánchez, J. y Morales, J. (2012). La inferencia en la comprensión de textos expositivos de ciencias sociales. Enunciación, 17 (2), 3-17. 
Solé, I. (1992). Estrategias de lectura. Barcelona: Graó. Spire, A. (1999). Sobre la fenomenología. Entrevista a Jacques Derrida. En Staccato, programa de televisión de France Culturel.

Van Manen, M. (2003). Investigación educativa y experiencia vivida. Ciencia humana para una pedagogía de la acción y de la sensibilidad. Barcelona: Idea Books.

4 International Colleges \& Universities (2014). Universidad de Bogotá Jorge Tadeo Lozano. Recuperado de http://www.4icu.org/reviews/1054. htm 